\title{
Conceptual and Practical Meaning of Technique in Running Performance - a brief observation of the problem
}

\author{
Nikolay Romanov* \\ Pose Method, Miami FL, USA
}

\begin{abstract}
The article is addressing the problem of running technique as a major factor of improving running performance. A short retrospective review showed that the main obstacle in developing a proper and efficient running technique was the absence of a commonly accepted concept, model of the efficient running technique, which, in its turn, did not allow the development of an efficient method of teaching. The author, based on his own studies in this field, is proposing a very specific way of solving this problem by using gravity as the major source of running movement with the rest of the factors just assisting it.
\end{abstract}

\section{Introduction}

One of the main reasons of why people run, beside the desire to get healthy, happy, lose weight, get fun, is to challenge themselves and run faster to fulfill their potential. Most runners want to improve their performance and they look for all possible sources to make this happen. On the surface, the answer is seemingly simple and well-known. In order to run faster an athlete should be injury-free, dedicated and devoted to training hard, with high mileage, proper nutrition, right shoes, strength and stretching exercises, etc. There has never been any shortage of advice in this field in books, magazines and Internet articles, but one thing has always been almost missing - running technique. Two problems were always related to this - the absence of a commonly accepted running technique model and an adequate method of teaching, based on this model.

\section{Materials and methods}

Despite the fact that "Human gait... has always been in the forefront of biomechanical study" [1], and "coaches and athletes are forever arguing about the "ideal technique" [2], any consensus, of what to consider a proper running technique, has never been reached. In his concluding remarks to the book of "Biomechanics of Distance Running", [1] editor Peter Cavanagh came to a clear conclusion, "When reviewing the history presented here, it is hard to escape the verdict that despite the tremendous advances in computing power and instrumentation that have occurred in the last 20 years, we are basically still trying to answer many of the questions posed by Fisher and Fenn 90 and 50 years ago respectively."

That uncertainty in the vision on running technique, professor Peter Cavanagh expressed again many years later in 2008 in his interview for the New York Times ${ }^{(3)}$, - "The notion that there is one way to run is not, in my opinion, correct," he said and continued, - "Everything people are told is based on the best opinion of coaches rather than on the best evidence." This is quite a hard verdict acknowledging one obvious thing - science in running was still at the descriptive stage of data collections, without a sound theory of this motion, and this problem is still at the same stage nowadays.

There are lots of opinions, points of view, answers, "explanations", "models", and "theories" on running technique, produced over the course of the last century, by scientists and coaches, and athletes. Their range of thoughts could be described as insightful, good, bad, and sometimes, probably, nonsensical. It was quite an interesting mix of myths and reality. But in general, our conclusion is that there is no consensus, so far, neither in the science, nor coaching communities, on what to consider the right, proper, optimal running technique. Consequently, in order to understand the situation in this field better, below is presented a historical outline of running technique quotations, made by the leading scientists over the 70 years' period. This will allow us to see how this problem evolved and progressed.

For example, back in 1937, the founder of biomechanics in the Soviet Union, professor N. Bernstein wrote ${ }^{(4)}$, "Running technique, its physiological and dynamical structure from long time ago attracted attention of researches, however, having

\footnotetext{
${ }^{*}$ Corresponding author: pianzin@ mail.ru
} 
overwhelming opinions on this subject, nevertheless, left very little known about how exactly running is happening and what is its internal, refined (delicate) dynamic," - indicating the problem from the beginning. His point of view is showing the controversy related to running technique: various opinions and no definite knowledge about it.

Thirty years later, the famous British sport biomechanist, Geoffrey H.G. Dayson said almost the same ${ }^{3}$, "Running, «the classical athletics sport», can be considered both simple and difficult: simple, because it is an instinctive, natural skill performed at the same time by all but the most unfortunate; difficult in its mechanical complicity" [5], highlighting again the same controversy in the nature of running movement, but not proposing any specific concept leading us to a deep understanding of running motion.

Well after almost another twenty years, another scientist from the Soviet Union, professor V. Nazarov wrote [6], "we are facing a paradox: there are overwhelming facts available, discussing details of movement, but still no fundamental mechanism found, by which a man is moving over the hard surface". As it was stated before, it was again about a conflict between a lot of data and the absence of a unifying theory and concept, allowing us to put all the data under one logical roof, with some general denominator explaining the nature of running motion.

As professor Bernard Heinrich wrote in his book [7] in 1995, "for millions of years, our ultimate form of locomotion was running. We are, deep down, still runners, whether or not we declare it by our action." The truth about running, he continues, is that while "... it may be simple in its design, it is awesomely complex in its details. Perhaps running, unlike weight events, involves relatively little restructuring of the body from what it is designed to do already, given the genetic raw material, proper nutrition, and a few simple instructions. The question is, what is the raw material and what are the environmental triggers, or "instructions?" Indeed, what are they? It seems to be a simple thing, but not easy to understand.

In August 2014 at Calgary International Running Symposium Dr. John E. A. Bertram, made an essential statement in his presentation [8], "it is observed that normal running is remarkably well described, but is not well understood. Although we can fully characterize how running is accomplished, we do not know why it is accomplished as it is", which brings us back to one and the same conclusion, that running science has never got to a conceptual level, allowing us to explain how running is happening. The sad consequences of that situation go to all aspects of running, including injury prevention and healing, teaching and training efficiency (identification of errors and their correction, running economy etc.), racing performance and so on.

At the same Calgary University symposium, the author of this article had an opportunity as a speaker at the panel "Injuries in Running and Minimalistic Shoes" to talk about a real epidemic of injuries, the running community (coaches, scientists, athletes) have been facing over the last few decades. Neither shoes development by footwear companies, nor science studies, nor coaches' efforts, were helpful enough to reduce the incidents of injuries. On the opposite, over last forty years, the injury rate was growing from $65 \%$ at the end of 1970 s to $85 \%$ at the present time.

At this short presentation, the author defined the causes of the problem as stemming from the absence of the standard of a proper running technique, a sound biomechanical concept and a model of running movement, which, in addition, were leading to the absence of the efficient method of teaching the running technique. He also proposed to unite the efforts of all parts of the running community for development of the consensus on what to consider a proper running movement (technique), its model and standards in order to solve this long-lasting problem and therefore establish a new paradigm of running. Unfortunately, that proposal didn't find much support among the scientists of this symposium. It seemed the science community at that time wasn't ready to move to the new paradigm.

If we look back to the time when the running boom started, we can find out that running technique wasn't a priority. The legendary running coach and cofounder of Nike, Bill Bowerman, was very opinionated on this matter in his book "Jogging" [9] - "Running is a simple kind of exercise requiring no highly developed skills. Do it, and don't worry about trying to look right." He, basically, was blessing running ignorance for the next several decades, which caused an epidemic high injury rates in runners.

This line of thoughts didn't change almost until present time. Gina Kolata in her 2008 article [3] in The New York Times wrote, "Great swimmers must have great genetic gifts and also a great technique. The same goes for pole-vaulters and high jumpers, for tennis players and gymnasts. But the men who are running in the marathon here Sunday will hardly exemplify one perfect technique." Basically, the author was reflecting a common vision on running technique by scientists, coaches and the whole running community.

Therefore, due to the lack of understanding of the role of running technique, the major emphasis in training was put on the development of physiology, or aerobic capacity.

So, what was left for runners to do to improve their results? Work hard! Running technique and skill has never been a topic for a serious consideration in training. However, the last decade was the time when the situation gradually has been turned around and the new understanding of the value of technique for improving running performance was developed [10, $11,12,13]$. The author of one of the articles ${ }^{(14)}$ was writing that, "The 'one best way' isn't about footwear. It's about form. Learn to run gently, and you can wear anything."

Most recent publication ${ }^{(15)}$ is openly expressing that, "Until a few years ago, the accepted wisdom for those looking to improve performance was just to run more, train smarter, or lose weight. Now, you might have to rethink how you run". This article describes a story of a runner Blue Benadum, who ran 35 marathons before changing his form because, no matter the efforts and different programs, he could never break 2:42. Then he took a VO2-max test to gain some insight into his 
fitness potential. He got an $85 \mathrm{ml} / \mathrm{kg} / \mathrm{min}$, the highest score, which put him in the top rank of performers. This insight brought him to the decision to change his running form. After a year of working on his form, times started to progress: 2:38, $2: 35,2: 28,2: 24$, and 2:23. It makes sense to look at a related story of Lance Armstrong attending the New York City marathon. As is well known, Lance Armstrong had one of the highest $\mathrm{VO}_{2} \max (84 \mathrm{ml} / \mathrm{kg} / \mathrm{min})$, but his marathon performance, unlike as his cycling racing, was pretty average and he got just slightly under the 3 hours and after the race he sustained painful conditions in his legs. The latter was just a confirmation of his poor running technique.

\section{Results and discussion}

This shift in paradigm was a logical consequence of the failure of previous decades to solve injuries problems and stagnation in performance of many runners around the world.

Nowadays, this line of thinking lead to understanding that running is something you have to learn. The task of getting faster was not so much about how you trained, but how you ran. In other words, it was about running technique. On the surface, when the elite runners were observed, it was clear that their superiority lied not only in their physiological and physical condition, providing for their high-level performance, but also in their movement. The usual description of it would use visual characteristics such as effortless, smooth, light, short support running. For example, the following was a commonly accepted visual description of running of the legendary Emil Zatopek [14], "from the waist down, Zatopek was always quick, light and springy", but what was behind this wasn't clear. Nevertheless, the necessity and desire to look for the "best way"

My endeavor in this field took off four decades ago with the idea that running is no different from any other movement and requires a technique or skill of doing, as it is translated from Greek [10]. Understanding, that skill is the ability to use all available resources to reach the goal, may help us to find the right approach to this problem. Everything we learn, we adapt to ourselves and then express in our performance. In other words, everything we get in and out of our organism must have its own entrance, and exit - the gate. How we move in running shows our interaction with our environment and may be proper or wrong, which has a direct reflection on our performance. The essence of this interaction is how we use gravity in our movement [16].

What was never understood in general on this matter is a hierarchy of parameters providing for an athlete's running performance. Conventional wisdom and science were putting physiological parameters in distance running as a major limiting factor $\left(\mathrm{VO}_{2}\right.$ max, economy, anaerobic threshold), with almost no significant value attributed to running technique, which generally was one of the most significant factors of improving performance, though never seen and understood like that, due to the lack of related concepts. According to Jack Daniels's [16] opinion, shared by many, it sounds as following: "Whatever you run heel - toe, toe - heel if it fits you, it's okay". The conclusion from such an ambiguous opinion - is that nature doesn't care how you are interacting with it.

Obviously, our physiology is playing an essential role in endurance in running, but the speed of running, from our conceptual point of view, comes from technique (the skill of doing). To make it clearer, it is about how much we are utilizing the force of gravity. From the results of our studies $[16,18,19]$ we came to the conclusion that the speed of running directly depends on the angle of falling of the runner during the second half of the support phase. We can compare, with some approximation, in this case, physiological and mechanical capacity of a runner with a car, where an engine defines the speed of the car and size of the gas tank defines how long it could drive. So, the engine of the runner is his falling angle, and the tank is his aerobic capacity. For the simplicity's sake, we can avoid, at this point, any other components of performance such as his mental capacity, muscular morphology etc., which are playing their own important, but subservient roles, in the runner's performance.

\section{Conclusions}

So, for the runner, with all these components of capacity - biomechanical, physiological, psychological, mental and spiritual - all existing simultaneously in space and time, the final result in performance comes through the mechanical gate, which is his skill to execute a bigger angle of falling of the body on the support, to increase the horizontal transfer of the runner. This final requirement is the most distinctive characteristic ${ }^{(18)}$ of the top runners. Our research on this matter directly confirmed this statement with regards to the performance of athletes in sprinting and distance running.

When it comes, for example, to Usain Bolts performance ${ }^{(19)}$, we found that his most advantageous characteristic is a bigger angle of falling (21.4-21.6 $)$, compared to his competitors, who had much higher stride cadence (in the best 9.58 sec $100 \mathrm{~m}$ World Record run he had the cadence of 256 steps per minute, while Tyson Gay had 281 steps per minute in the same final in Berlin World Championship in 2009). The same logic holds true in the recent performance of the World Record holder on 400m in Rio de Janeiro Olympic games, Wayne Van Niekerk. His 43.03 seconds came with a bigger average angle of falling $\left(20.41^{0}\right)$, compared to the previous one (43.18) by Michael Johnson $\left(18.87^{0}\right)$.

This essential reality holds true across all running, including distance running, where the progress in marathon from Haile Gebrsilassie to Eliud Kipchoge records depended on the angle of falling, with some regard to step cadence, and some other factors comprising elite performance. Having said that, I still want to stress, that the angle of falling, as the way of 
utilizing gravity, should be counted as the major contributing factor to improving running performance. When this factor is not utilized, other factors, such as step cadence, can't compensate fully for the absence of the first one.

This is the reason that so many runners can't progress, no matter their dedication, hard work, high volume of training, etc. Therefore, runners training, in order to continue improvement of their performance, must follow this hierarchical logic through the time of their carriers. Learning how to run must be a process of developing the skill of falling in the frame of the Running Pose and utilizing the action of Pulling the foot from the ground for recovering Pose at every single step. That approach to training in running as the development of the skill of falling, puts all the components of training for different distances in their proper perspective regarding the main factor - the falling angle.

\section{References}

1. P.R. Cavanagh, Biomechanics of Distance Running (Human Kinetics Books, Champaign, Illinois, 12, 31, 1990)

2. H. Payne, Athletes in Action: the official International Amateur Athletic Federation (IAAF) book of track and field techniques (Editor Pelham Books, 9, 1985)

3. Kolata G. There Are No Points for Style in the Marathon (New York Times, 2008).

4. N.A. Bernstein, Theory and practice of physical culture, 250-261 (1937)

5. G.H.G. Dyson, The Mechanics of Athletics (University of London Press, 109, 1967)

6. A.I. Pyanzin, Pedagogico-psychological and medico-biological problems of physical culture and sports, 11(3), 118-125 (2016)

7. J. Bertram, International Calgary Running Symposium, August 14-17 (2014)

8. W. Bowerman, W.E. Harris, Jogging. Grosset and Dunlap (New York, 7, 1967)

9. N. Romanov, Pose Method of Running (Pose Tech Press, 2002)

10. R. Wallack, Runner's World, 109, 68-73 (2004)

11. C. McDougall Born to Run (Vintage Books, New York, 2009)

12. J. Kahn The perfect stride (New Yorker, November 8, 2010)

13. T. Parker-Pope The One Best Way to Run (November 2, 2011). URL: http://well.blogs.ntimes.com/tag/marathon/

14. M.F. Huber, The Outside Magazine (February 15, 2016)

15. N. Romanov, G. Fletcher, International Journal of Sports Biomechanics, 6(3), 434-452 (2016)

16. J. Daniels, Daniels' Running Formula (Champaign, IL, Human Kinetics, 80-82, 1998)

17. N.S. Romanov, A.I. Pianzin, Book of Abstracts of the 11th Annual Congress of the European College of Sport Science (Lausanne, $582,2006)$

18. N. Romanov, Distinctive Characteristics of Usain Bolt's Running Technique (Posetech, 2009) 